\title{
Use of hESC Lines by CIRM Grantees: The Value of Embryonic Stem Cell Research Oversight (ESCRO) Committees
}

\section{Rohun Patel and Geoffrey Lomax*}

California Institute for Regenerative Medicine, San Francisco, California 94107, USA

\section{Introduction}

Human embryonic stem cell (hESC) research occupies an exceptional place in science because governments and funding organizations have adopted unique polices to influence research conduct [1]. These policies often extend to the research tools themselves, hESC lines. For example, the U.S. NIH maintains a registry of hESC lines approved for use in research it funds. The U.K. Stem Cell Bank employs a vetting procedure to ensure deposited lines conform to specific requirements.

As a consequence the use of specific hESC lines is a topic of ongoing international interest because utilization patterns serve as a research and evaluation tool. For example, previous studies have examined utilization patterns to evaluate the impact of state-based research programs [2]. McCormic et. al. examined distribution rates and patterns of hESC line utilization as an indicator of state research capacity [3]. Karmali et al. [4] examined the use of hESC lines not eligible for NIH funding as an indicator of the efficacy of state-funded stem cell research programs [4]. These studies quantified transfers of selected hESC lines or reported surveys of hESC line requests as indicators or proxies to support their analysis.

In this article, we build on this literature by quantifying the utilization of specific hESC lines based on reports from CIRM grantees. These reports provide an added level of resolution allowing us to (1) elaborate on findings from previous studies and (2) evaluate the efficacy of CIRM-specific policies for research review and oversight.

\section{CIRM policies for research review and oversight}

The California Institute for Regenerative Medicine (CIRM) was established, in part, to support research not eligible for federal funding in the United States. In 2006, the Institute promulgated regulations consistent with the National Academies' Guidelines for Human Embryonic Stem Cell Research. The NAS Guidelines are intended to support the responsible and ethical conduct of research not eligible for federal funding [5]. The guidelines recommend institutions establish human embryonic stem research oversight (ESCRO) committees to provide oversight for the use of hESC lines not otherwise covered by the NIH guidelines.

CIRM has incorporated the NAS Guidelines as formal regulations applicable to all CIRM-funded research. The CIRM regulations allow grantees to utilize human embryonic stem cell lines that conform to standards for acceptable derivation [6].

${ }^{1}$ Lines derived in accordance with the Japanese National guidelines, Canadian Institutes for Health Research guidelines, UK Human Fertilization and Embryology Authority (UK HFEA) license or Australian Research Involving Human Embryos Act license are also acceptable for use. However, no lines direved in accordance with the Japanese, Canadian or Australian policies were utilized by CIRM grantees and UK HFEA lines were also deposited in the UKSCB.
To be acceptably derived any one of the following criteria must be met:

1. Be approved for use in NIH-funded research after March 2009 (NIH Registry Lines) [http://stemcells.nih.gov/research/ registry/]

2. Be derived by a CIRM-funded grantee in accordance with CIRM requirements (CIRM-derived lines) [http://www.cirm. ca.gov/files/reg/pdf/Reg100080_SM_Acct_Standards.pdf]

3. Be determined to meet CIRM standards by anESCRO committee (ESCRO approved lines)

4. Be NIH-approved prior to 2009 but not currently listed in the NIH Registry (NIH Registry prior to 2009) [http:// stemcells.nih.gov/staticresources/research/registry/PDFs/ FormerRegistry.pdf]

5. Be deposited in the UK Stem Cell Bank (UKSCB lines) [http:// www.ukstemcellbank.org.uk/stemcelllines.cfm]

CIRM grantees document their use of specific hESC lines in annual progress reports. Based on these reports, hESC lines may be classified according to the regulatory / policy criteria above. Criterion number three, ESCRO approved lines, is of particular interest with regard to evaluating the efficacy of policies for research review and oversight. CIRM and other state-based stem cell research programs require ESCRO oversight of most funded protocols. Further, ESCRO committees report hESC provenance review to be a major programmatic activity. For example, $75 \%(n=21 / 28)$ of ESCRO committees responding to a national survey by the Intestate Alliance on Stem Cell Research http:// iascr.org/docs/2011/ESCRO\%20committee\%20survey\%202011.pdf reported provenance review to be a responsibility. For purposes of evaluating policy efficacy, it is helpful to understand the role ESCROs play in enabling research that would otherwise be ineligible for federal funding.

\section{Methods}

CIRM's reporting forms request grantees to identify all hESC lines used in Institute-funded research during the previous year. Reporting

*Corresponding author: Geoffrey Lomax, California Institute for Regenerative Medicine, San Francisco, California 94107, USA, E-mail: glomax@cirm.ca.gov

Received October 14, 2011; Accepted November 12, 2011; Published November 14,2011

Citation: Patel R, Lomax G (2011) Use of hESC Lines by CIRM Grantees: The Value of Embryonic Stem Cell Research Oversight (ESCRO) Committees. J Stem Cell Res Ther 1:107. doi:10.4172/2157-7633.1000107

Copyright: (C) 2011 Patel R, et al. This is an open-access article distributed unde the terms of the Creative Commons Attribution License, which permits unrestricted use, distribution, and reproduction in any medium, provided the original author and source are credited. 
Citation: Patel R, Lomax G (2011) Use of hESC Lines by CIRM Grantees: The Value of Embryonic Stem Cell Research Oversight (ESCRO) Committees. J Stem Cell Res Ther 1:107. doi:10.4172/2157-7633.1000107

Page 2 of 3

is performed either by choosing from a list of 39 common hESC lines and/or by self-entering the names of lines not included on the list. As of August 2011, CIRM had 238 active awards that met the inclusion criteria for this analysis. To be included: (1) the grantee must have submitted one or more annual progress reports and (2) the award must be a research grant (training, planning and facilities grants were excluded). 97 grants (41\%) reported utilizing one or more hESC lines.

All hESC lines used in these 97 grants were then coded according to their regulatory status at the time of analysis. Any particular line could be included in one or more of the following regulatory categories: (1) Current NIH Registry Lines, (2) CIRM derived lines; (3) ESCRO approved lines, (4) NIH Registry prior to 2009, or (5) UKSCB lines.

Any particular line could be included in one or more regulatory category, but for reporting purposes, each was assigned to a unique category based on a hierarchy. Any given line was assigned to the lowest possible numerical category. For example, H9 was approved for use by NIH in 2001 (category 4), and it was subsequently approved for use after December 2009 (category 1). Therefore, H9 is assigned to category 1. Similarly, CIRM grantees have reported the derivation of 17 lines (category 2). Seven of these lines are NIH registered, so they were assigned to category 1 . This hierarchical approach allows us to distinguish between research eligible for federal funding, predominantly NIH.

\section{Results}

138 unique hESC lines were utilized in the 97 grants meeting our inclusion criteria. Seventy-five percent of the hESC lines utilized $(n=103)$ were not eligible for federal funding at the time this analysis was conducted - regulatory status 2-5. Table 1 reports the utilization of lines according to their current regulatory status.

The cross-sectional nature of this analysis should be recognized. For example, grants involving the ten lines in category 4 (NIH Registry prior to 2009) were awarded prior to the Presidential Executive Order of March 2009. Therefore, they would have been eligible for federal funding at the time of the award. If one were to consider eligibility at time of funding then, sixty-seven percent of the lines utilized were ineligible.

The majority of lines used $(n=70)$ by CIRM grantees were determined to meet CIRM standards by an ESCRO committee. This figure is double the number of currently NIH-eligible lines being used. In addition, sixteen of the hESC lines used were derived and utilized by CIRM grantees. Of these sixteen, seven are included in the current NIH registry (Table 2).

In terms of intensity of utilization, $\mathrm{H} 9$ and $\mathrm{H} 1$ are the most

\begin{tabular}{|l|c|c|}
\hline Regulatory Status & Number of Lines & Percent \\
\hline [1] Current NIH Registry lines & 35 & 25 \\
\hline $\begin{array}{l}\text { [2] CIRM derived Lines (not in current NIH } \\
\text { Registry) }\end{array}$ & 9 & 7 \\
\hline [3] ESCRO approved lines & 70 & 51 \\
\hline [4] NIH Registry prior to 2009 & 10 & 7 \\
\hline [5] UKSCB lines & 14 & 10 \\
\hline Total: & 138 & 100 \\
\hline
\end{tabular}

Table 1: CIRM Grantee hESC Utilization by Regulatory Status.

\begin{tabular}{|c|c|c|}
\hline $\begin{array}{c}\text { Line } \\
\text { Name }\end{array}$ & $\begin{array}{c}\text { Number of } \\
\text { Grants }\end{array}$ & $\begin{array}{c}\text { NIH } \\
\text { Approved }\end{array}$ \\
\hline H9 & 77 & Yes \\
\hline H1 & 53 & Yes \\
\hline HuES-9 & 17 & Yes \\
\hline H7 & 15 & Yes \\
\hline HSF1 & 13 & No \\
\hline HSF6 & 11 & No \\
\hline HuES-6 & 11 & Yes \\
\hline HuES-7 & 10 & Yes \\
\hline HuES-1 & 8 & Yes \\
\hline HES 2 & 7 & No \\
\hline
\end{tabular}

Table 2: hESC Lines Most Frequently Used by CIRM Grantees.

frequently utilized lines involved in 77 and 53 studies respectively. Current NIH registry lines account for seven of the top ten lines utilized.

\section{Discussion}

Consistent with previous studies, hESC lines derived at WiCell Research Institute (H series) and Harvard University (HuES) are the most frequently utilized. This intensity of utilization may be attribute to their NIH eligibility status, well developed material transfer agreements, policy decisions to support their distribution through the National Stem Cell Bank and their resulting scientific utility.

We also observe an ongoing research investment in hESC lines approved by NIH prior to 2009 but not currently listed in their Registry. These lines include HSF1, HSF 6 and HES1-5 used in 13, 11 and 20 protocols respectively. Under NIH policy, grantees may continue to utilize lines in grants awarded before March 2009, but such use would eventually be phased out. CIRM policy allows for the use of all current and formerly registered NIH lines. We believe this approach serves to support research programs that have made a sizable investment in lines approved under the former policy.

$75 \%$ of hESC lines utilized by CIRM-funded researchers are not eligible for use in NIH-funded studies. Due to their timing and methodological limitations, previously cited studies were not able to quantify this proportion. In addition, CIRM-funded researchers derived $17 \mathrm{hESC}$ lines. These data suggest state-based research programs serve to expand the scope of studies and the supply of hESC lines.

The data also illustrate the importance of embryonic stem cell research oversight (ESCRO) committees in evaluating the provenance of hESC lines. The majority of hESC lines utilized by CIRM grantees are not currently or previously approved by NIH or deposited in the UKSCB. Such lines must be evaluated against criteria consistent with the National Academies' Guidelines for Human Embryonic Stem Cell Research. Given there are over 1000 lines (ISSCR accessed 09/07/2011) identified in the UMASS International Stem Cell Registry, there is an ongoing need to evaluate the provenance of hESC lines to ensure they are derived according to consensus standards.

The ability of researchers to utilize ESCRO committees is critical for allowing immediate access to lines not otherwise approved by other regulatory bodies. CIRM-funded researchers have suggested this access serves to fill knowledge gaps in the field of human pluripotent cell biology. 
Citation: Patel R, Lomax G (2011) Use of hESC Lines by CIRM Grantees: The Value of Embryonic Stem Cell Research Oversight (ESCRO) Committees. J Stem Cell Res Ther 1:107. doi:10.4172/2157-7633.1000107

Page 3 of 3

\section{Acknowledgements}

We thank Bill Gimbel, Kelly Shepard and Steve Torrence for their efforts to provide the underlying data to support this analysis.

\section{References}

1. Lomax G, McNab A (2008) Harmonizing standards and coding for hESC research. Cell Stem Cell 2: 201-202.

2. Levine AD (2011) Policy uncertainty and the conduct of stem cell research. Cell Stem Cell 8: 132-135.

3. McCormick JB, Owen-Smith J, Scott CT (2009) Distribution of human embryonic stem cell lines: who, when, and where. Cell Stem Cell 4: 107-110.

4. Karmali RN, Jones NM, Levine AD (2010) Tracking and assessing the rise of state-funded stem cell research. Nat Biotechnol 28: 1246-1248.

5. NAS (2010) Final report of the National Academies human embryonic stem cel research advisory committee and 2010 amendments to the National Academies' Guidelines for Human Embryonic Stem Cell Research. Washington, D.C., National Research Council (U.S.).

6. Lomax GP, Hall ZW, Lo B (2007) Responsible oversight of human stem cel research: the California Institute for Regenerative Medicine's medical and ethical standards. PLoS Med 4: e114. 